\title{
Serious Gaming for Test \& Evaluation of Clean-Slate (Ab Initio) National Airspace System (NAS) Designs
}

\author{
B. Danette Allen ${ }^{1}$ and Natalia Alexandrov ${ }^{2}$ \\ NASA Langley Research Center, Hampton, VA, 23681
}

\begin{abstract}
Incremental approaches to air transportation system development inherit current architectural constraints, which, in turn, place hard bounds on system capacity, efficiency of performance, and complexity. To enable airspace operations of the future, a clean-slate (ab initio) airspace design(s) must be considered. This ab initio National Airspace System (NAS) must be capable of accommodating increased traffic density, a broader diversity of aircraft, and on-demand mobility. System and subsystem designs should scale to accommodate the inevitable demand for airspace services that include large numbers of autonomous Unmanned Aerial Vehicles and a paradigm shift in general aviation (e.g., personal air vehicles) in addition to more traditional aerial vehicles such as commercial jetliners and weather balloons. The complex and adaptive nature of $a b$ initio designs for the future NAS requires new approaches to validation, adding a significant physical experimentation component to analytical and simulation tools. In addition to software modeling and simulation, the ability to exercise system solutions in a flight environment will be an essential aspect of validation. The NASA Langley Research Center (LaRC) Autonomy Incubator seeks to develop a flight simulation infrastructure for ab initio modeling and simulation that assumes no specific NAS architecture and models vehicle-tovehicle behavior to examine interactions and emergent behaviors among hundreds of intelligent aerial agents exhibiting collaborative, cooperative, coordinative, selfish, and malicious behaviors. The air transportation system of the future will be a complex adaptive system (CAS) characterized by complex and sometimes unpredictable (or unpredicted) behaviors that result from temporal and spatial interactions among large numbers of participants. A CAS not only evolves with a changing environment and adapts to it, it is closely coupled to all systems that constitute the environment. Thus, the ecosystem that contains the system and other systems evolves with the CAS as well. The effects of the emerging adaptation and co-evolution are difficult to capture with only combined mathematical and computational experimentation. Therefore, an ab initio flight simulation environment must accommodate individual vehicles, groups of self-organizing vehicles, and large-scale infrastructure behavior. Inspired by Massively Multiplayer Online Role Playing Games (MMORPG) and Serious Gaming, the proposed ab initio simulation environment is similar to online gaming environments in which player participants interact with each other, affect their environment, and expect the simulation to persist and change regardless of any individual player's active participation.
\end{abstract}

${ }^{1}$ NASA Senior technologist (ST) for Intelligent Flight Systems (IFS), Head of NASA LaRC Autonomy Incubator (AI), MS 492, AIAA Senior Member.

${ }^{2}$ Lead, Ab Initio Design of Autonomous Airspace Operations, NASA LaRC

Aeronautics Systems Analysis Branch, MS 442, AIAA Associate Fellow. 


\section{Nomenclature and Acronyms}

$\begin{array}{ll}\text { AEON } & \text { Autonomous Entity Operations Network } \\ \text { AI } & \text { Autonomy Incubator } \\ \text { ALHAT } & \text { Autonomous Landing and Hazard Avoidance Technology } \\ \text { ARES } & \text { Aerial Reconfigurable Embedded System } \\ A T M & \text { Air Traffic Management } \\ C A S & \text { Complex Adaptive System } \\ C E R T A I N & \text { City Environment for Range Testing of Autonomous Integrated Navigation } \\ C O A & \text { Certificate of Waiver or Authorization } \\ \text { DDS } & \text { Data Distribution Service } \\ H I T L & \text { Human-In-The-Loop } \\ I F S & \text { Intelligent Flight Systems } \\ \text { LaRC } & \text { Langley Research Center } \\ M M O R P G & \text { Massively Multiplayer Online Role Playing Game } \\ N A S & \text { National Airspace System } \\ O O D A & \text { Observe Orient Decide Act } \\ P I & \text { Principle Investigator } \\ R \& D & \text { Research \& Development } \\ R O S & \text { Robot Operating System } \\ S A & \text { Situation Awareness } \\ U A S & \text { Unmanned Aerial System }\end{array}$

\section{Introduction}

$\mathrm{T}$ HE Autonomy Incubator ${ }^{1}$ (AI) at NASA Langley Research Center (LaRC) was established in the spring of 2014 to prepare the Center workforce to meet the autonomy challenges that are anticipated in science, space exploration, and aeronautics as the NASA mission directorates seek to enable new missions such as asteroid retrieval, planetary exploration, atmospheric sensing in historically inaccessible areas, and the integration of Unmanned Aerial Systems (UAS) into our everyday lives - all missions of increasing complexity, distance, proximity, pace, and/or accessibility. Building on decades of experience and success in the design, fabrication, and integration of safe and reliable automated systems for space and aeronautics, the NASA seeks to bridge the chasm between automation and autonomy and build intelligent systems that are capable of

1. OBSERVING - sensing and perceiving their environments

2. ORIENTING - assessing their state and situation awareness (SA)

3. DECIDING - making decisions in the face of uncertainty and/or with incomplete information

4. ACTING - executing those decisions

These autonomous intelligent systems will be adaptive in their response to unforeseen conditions, much the same way that humans are. They will intelligently execute the OODA loop ${ }^{2}$ (Observe-Orient-Decide-Act) and, ultimately, will extend OODA to OODAL with

5. LEARNING from experience and adapting to improve performance and robustness.

Autonomous mobility can be decomposed into a number of fundamental tasks ${ }^{3}$. Assuming that a mission plan is available, an intelligent agent in the system must be able to:

- Estimate its (ownship) state

- $\quad$ Sense and perceive its world

- Maintain and update its "map" of the world

- $\quad$ Fuse information from multiple sensor sources

- Detect and avoid objects/hazards

- Classify objects as benign or hazardous

- $\quad(\mathrm{Re})$ Plan its trajectory and path

- Execute inner loop control for agile flight.

In the same way that human pilots are responsible for the safety of humans in their aircraft, these intelligent flight systems (IFS) will be responsible for their own safety or, more accurately, their survivability ${ }^{4}$. Beyond the individual aircraft, the air transportation system itself is an IFS - complex and adaptive exhibiting emergent behaviors that result 
from interactions among its large number of participants. The AI has recently accepted a new challenge, building upon and extending beyond integrating UAS into today's National Airspace System (NAS) ${ }^{5}-$ Ab Initio NAS Design.

\section{The Air Transportation Complexity Challenge}

Large technological systems, such as the air transportation system and the Internet, were not designed as the systems we know today. They evolved organically and incrementally, in response to stimuli such as sprawling populations, insufficient throughput, and collisions. Reactions to accidents have been especially instrumental in advancing the technical development of the air transportation system. However, the incremental evolutionary approach to improving the air transportation system no longer suffices in the face of growing demand and the changing nature of airspace. The present air transportation system consists of a complicated set of interacting layers. All effort in ensuring its safety can be viewed as an attempt to prevent a merely complicated system from becoming an intractably complex one. That is, unavoidably, the current system consists of a large number of participants or agents, but safe control of the system relies on bounding the system complexity. This is accomplished via human-based, centralized control and partitioning of the system into manageable and controllable subsets (e.g., sectors). Although the National Airspace System (NAS) is subject to occasional, usually weather-related cascading failures, such phenomena are well understood. The top-down sytem control prevents true emergence. This is why the present-day sophisticated, high-fidelity airspace simulations (e.g., ACES, ATOS) represent the NAS faithfully enough to yield good predictions of the system's behavior in response to small perturbations. Simulations cannot guarantee a real system's behavior, but the current high-fidelity simulations serve as a reasonable tool for validation of new airspace processes if they do not deviate a great deal from the present ones.

With the rapidly changing nature of airspace participants and, in particular, the advent of autonomous and unmanned vehicles, the air transportation system of the future will be a complex adaptive system, or CAS. CAS and the phenomenon of emergence are determined by bottom-up processes, such as operation on autonomous vehicles. Simulations faithful to the current system will no longer suffice to serve as predictors of an airspace CAS behavior ${ }^{6,7}$ characterized by complex and sometimes unpredictable (or unpredicted) behaviors that result from frequently nonlinear temporal and spatial interactions among large numbers of participants. Traditional simulations view the system and its environment as distinct from each other: the system is stressed by environmental inputs. The salient characteristic of CAS is its lack of separation from the environment: a CAS not only evolves with a changing environment and adapts to it, it is closely coupled to all systems that constitute the environment. Thus, the ecosystem that contains the system and other systems evolves with the CAS as well. The effects of the emerging adaptation and co-evolution are difficult to capture with only combined mathematical and computational experimentation. Moreover, behavior prediction is complicated by strong dependence of CAS on initial conditions. This is why the future airspace CAS, characterized by a large degree of autonomy and hence distributed control, requires new approaches to validation, adding a significant physical experimentation component to analytical and simulation tools.

\section{Ab Initio Design}

The need to ensure airspace access for enormous numbers and variety of autonomous vehicles safely coexisting with independent, cooperative, and non-cooperative missions must be met. Human control places hard bounds on feasible directions available for incremental system development. Looking beyond incremental improvements in performance and towards the NAS as a complex adaptive system, the need for an $a b$ initio system design is apparent. ${ }^{13}$ To overcome the limitations of the current system and ensure safe operations for all participants in the national airspace system (NAS) of the future, we seek to design and implement a clean slate airspace design that can be mathematically modeled, simulated, and both analytically and empirically validated for survivability and resilience. In particular, we aim to:

- design aircraft explicitly for survivability and resilience in the environment of non-cooperative autonomy, without reliance of the external system and

- quantify how sensitive, aware, intelligent, adaptable, maneuverable, and thorough an individual aircraft must be to ensure the safety of its human passengers when the risk of collision grows with the presence of autonomous and unmanned aircraft.

The CAS that is the airspace of the future will be complex, crowded, cluttered and likely populated with heterogeneous vehicles piloted by both human and machine intelligence. Recently, NASA Research Announcements (NRA) and crowdsourcing $^{20}$ (Figure 1) were used to harvest ideas for overall airspace architectures, component concepts, and technologies that will enable the transition from today's NAS to the airspace of the future via clean-slate airspace architecture and operations, not constrained by the current system and restraints of the current transportation model. 
The proposed $\mathrm{Ab}$ Initio research aims to develop realistic vehicle requirements to design for resilience and survivability, in the presence of autonomy, based on vehicle capabilities, without reliance on an external system. In other words, what level of survivability can be achieved utilizing only ownship information and available data shared among NAS participants?

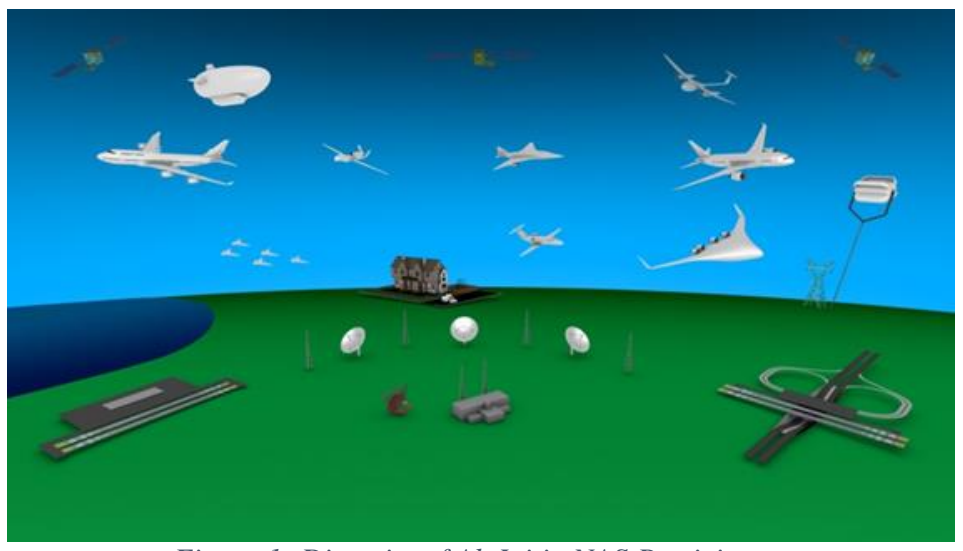

Figure 1: Diversity of Ab Initio NAS Participants

With this research goal comes a requirement for new simulation and test environments - environments that will accommodate plug-and-play participants with various "personalities", degrees of intelligence, and levels of capability for many thousands of "players", not unlike today's massively multiplayer online role-playing games. In addition to including human participant players in support of human-in-the-loop (HITL) evaluation, we can consider crowdsourcing the simulation and test as part of a broad evaluation approach. This approach falls into the category of serious games - simulations of real-world events created and conducted to solve real-world problems.

\section{Massively Multiplayer Serious Gaming for NAS Simulation}

A Massively Multiplayer Online Role Playing Game, or MMORPG, is a simulated world into which players (or, more generally, participants) can come and go while the simulated world persists. Popular examples of MMORPGs such as virtual world Second Life ${ }^{\mathrm{TM}}$ and the immensely popular World of Warcraft ${ }^{\mathrm{TM}}$, are rooted in tabletop games such as Dungeons \& Dragons ${ }^{\mathrm{TM}}$ (see Figure 2 ) $^{8}$. In the Autonomy Incubator, we plan to repurpose the characteristics of MMORPGs along with the enabling technologies to support persistent simulation of future designs of the NAS, the behavior of the player participants, individual or teamed goals, and function allocation between players and supporting infrastructure.
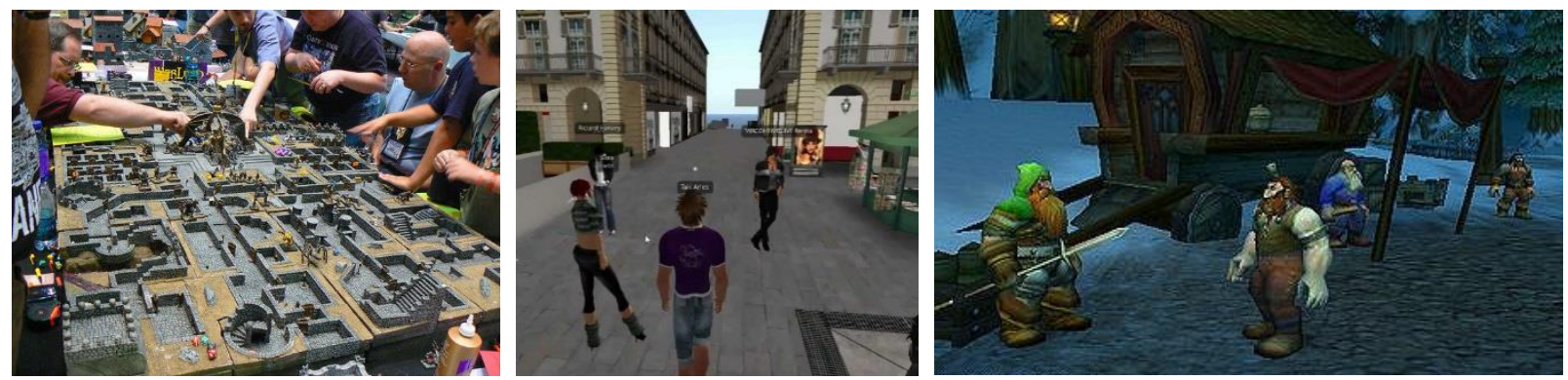

Figure 2: Evolution of Massively Multiplayer Games (left to right: Dungeons \& Dragons, Second Life, World of Warcraft)

A popular MMORPG game that is most closely related in functionality to the goal of simulating an Ab Initio NAS is Minecraft ${ }^{\mathrm{TM}}$. Developed in 2011, players can construct the environment, function in various "modes", and engage in activities or behaviors such as exploration, resource gathering, crafting, and combat. 

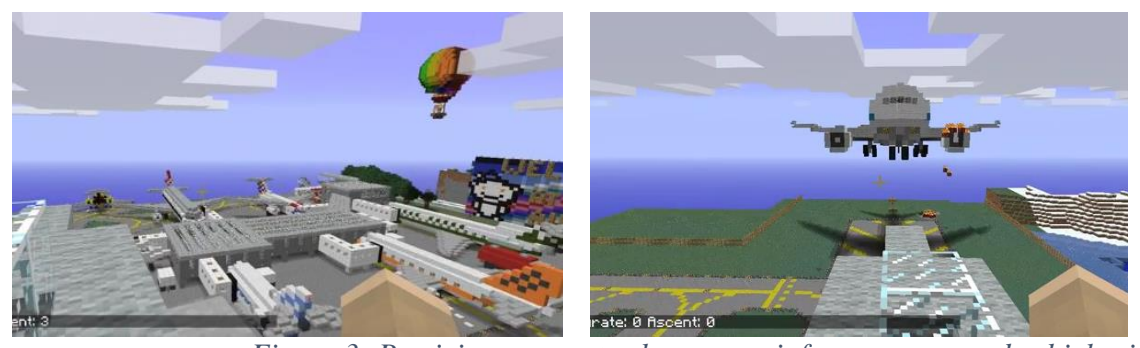

Figure 3: Participants create characters, infrastructure, and vehicles in Minecraft, ,,10

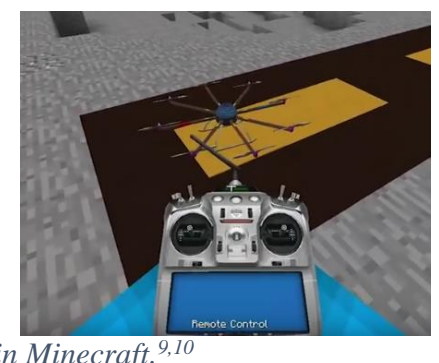

Minecraft ${ }^{\mathrm{TM}}$ is an example of an "open world" game in which player participants can move freely around in a persistent world, define their own goals or missions, and effect the simulation environment. As seen in Figure 3, there are already examples of NAS-related constructs and behaviors in Minecraft with a variety of aerial assets including balloons, commercial passenger jets, and drones. Any participant in a shared persistent world can interact with other participants and the environment.

\section{A. Persistent World}

A persistent world ${ }^{11}$ is a virtual world that "continues to exist and develop internally even when there are no people interacting with it". Typically, this refers to MMORPGs but also relates to "pervasive games" in which the real and virtual worlds are blended. This blending is not unlike the Live Virtual Constructive environments in use today for UAS in the NAS that allow simulation facilities from distributed locations to participate in a shared simulation environment. However, these environments are not pervasive and are not designed for exploring a solution space that includes innovative architectures, heterogeneous vehicles, changing "rules of the road", and player participants that enter and exit the simulation with their solutions at will. The intelligent flight systems created in the AI are capable of creating and maintaining world maps in real-time as well as transitioning between behavior states based on mission goals and execution. Transitioning from many distributed worlds to a shared centralized world that is predefined and modified in real-time is a natural extension of current capabilities.

\section{B. Plug-and-Play}

A critical characteristic of the proposed Ab Initio simulation environment is the ability to explore alternate methods and quickly integrate external solutions, which demands a software architecture and framework that supports collaboration, interoperability, and scalability. We have designed and implemented an open architecture that employs Data Distribution Service ${ }^{12}$, a DOD middleware standard for machine-to-machine communications.

\section{Open Architecture}

The AI's Autonomous Entity Operations Network (AEON) $)^{13}$ is an open distributed software architecture and a highly configurable data fusion framework that provides plug-and-play compatibility with a wide array of computer systems, sensors, software, and controls hardware. It also supports a ground control system(s) that acts as a test-bed for integration of multi-modal robotic vehicles. This architecture accommodates both onboard and offboard intelligence, allowing for live virtual constructive simulation of real and virtual assets, both local and remote.

\section{Middleware}

The AEON open architecture employs Data Distribution Service (DDS) for Real-Time Systems for messaging middleware. DDS is a publish-subscribe model (not unlike ROS, Robot Operating System ${ }^{14}$ ) to take advantage of "plug-and-play" network topologies, portability between systems, Quality of Service (QoS) guarantees between software entities, and an abstracted interface that external entities can meet without becoming experts in DDS. Further, DDS moves away from the idea of a "core" process and the associated risk of a single point of failure to a distributed entity-to-entity middleware approach. This distribution is an enabler for persistent game play and world modeling.

\section{Personalities}

Because we are emulating MMORPGs, human participants can insert themselves into the proposed simulation environment and assume any behavior(s) and goals(s) that they like. Not unlike alignments ${ }^{15}$ in Dungeons \& Dragons (a categorization of the ethical and moral perspective of player participants), virtual participating agents can assume one of five personality types: 


\section{- Collaborative}

Collaborative agents opt in and may even negotiate with other agents to optimize solutions. Collaboration may be system-wide or within a team of agents pursuing a common mission. Teammates may choose to sacrifice their objectives or themselves for the good of the mission or protect a teammate so that it survives an encounter.

\section{- Cooperative}

Cooperative agents are opting into the system with full disclosure about their state (6DOF position, intent, etc.) and may make use of services provided by the infrastructure. This is not unlike how we operate today under IFR conditions. Where coverage is available, there is two-way communication between aircraft and controllers, controllers to controllers, and even aircraft to aircraft.

\section{- Coordinative}

Coordinative (or non-cooperative) participants are friendly (mean no harm) while opting out of any possible data sharing options such as ADS-B, radar, etc. They will be invisible to any external system observing participating agents or providing services such as separation and collision avoidance.

\section{- Selfish}

Selfish participants are self-absorbed. They may have opted-in but are willing to ignore guidance and take risks that other participants would not. This is the equivalent of an aggressive driver that races from stoplight to stoplight and/or cuts off drivers when changing lanes. Their goal is not to cause harm but to maximize their performance and will do so at the expense of others if need be.

\section{- Malicious}

Malicious agents deliberately work against the system and the safety of its participants. They may opt in in terms of data but their shared information may be false or misleading.

Each personality type will be associated with a set of behaviors. Simulations can be run assuming various percentages/mixes of personality types and survivability of individual agents or types can be empirically observed. Survivability can be assessed in terms of ownship or an average based on the environmental composition. Independent variables may include but are not limited to:

- Traffic density

- Vehicle capability

$\circ$ Dynamics

$\circ$ Intelligence

- Data environment
○ Denied
- Degraded
- Deprived

- Personality mix (described above)

Further, virtual participants can be implemented as rule sets or as real humans interacting in a virtual NAS just as they would in the real NAS. These rule sets would be associated with a personality type as well as typical behaviors and aircraft performance characteristics.

Dependent variables will include but are not limited to separation distance, number of collisions, trajectory flexibility ${ }^{16}$, and, ultimately, survivability where survivability is defined as the probability of "either not being hit or, if hit, not crashing" $" 17$. 


\section{Flight Test and Evaluation}

Software simulation is an effective means of evaluating solutions over a range of independent variables with stochastic exploration and boundary conditions. However, simulation cannot capture all uncertainties especially the unpredicted behaviors. These "unknown unknowns" often reveal themselves only in physical tests - in flight. MultiUAV missions have been flown in the AI indoor flight area with as many as seven vehicles (Figure 4) and for various trajectory patterns including spiral for data collection or searching and loitering (Figure 5).

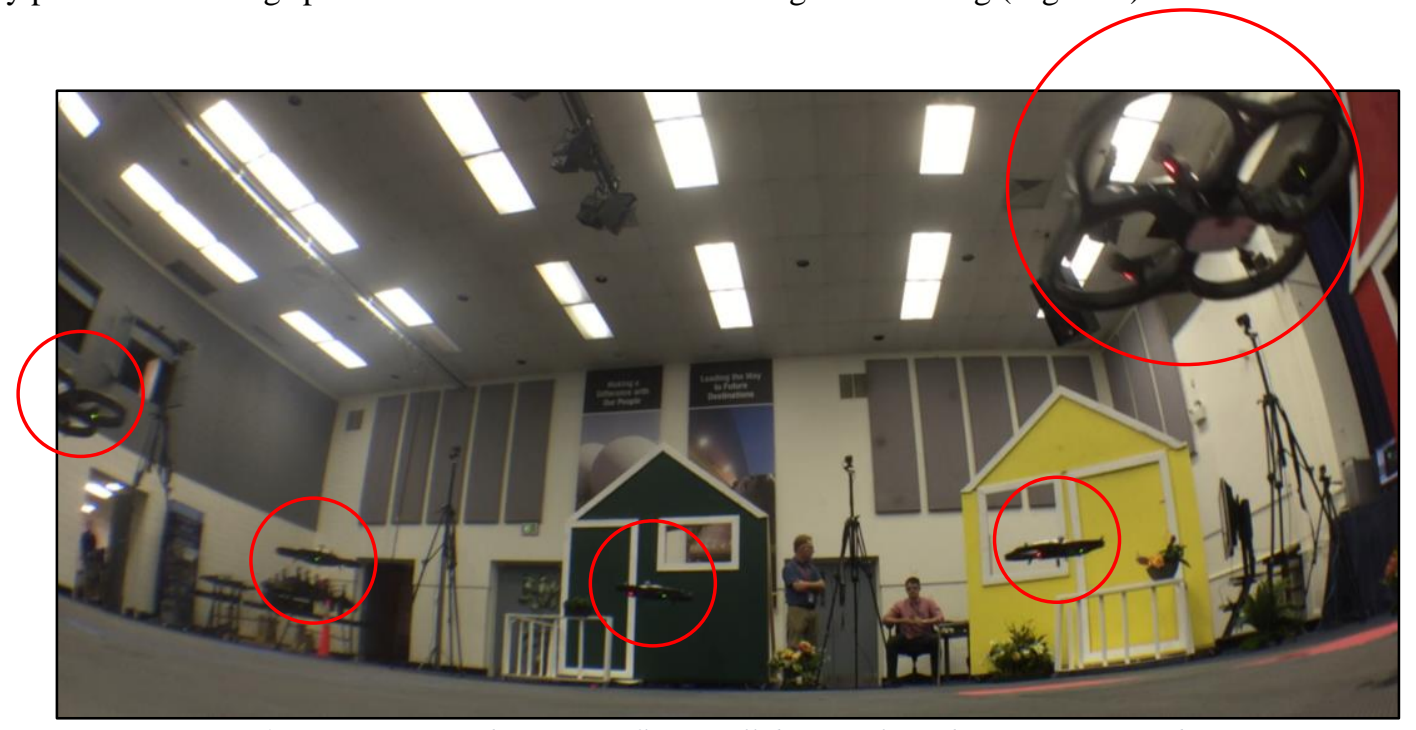

Figure 4: Five (n=5) machine assets flying collaboratively in the Autonomy Incubator

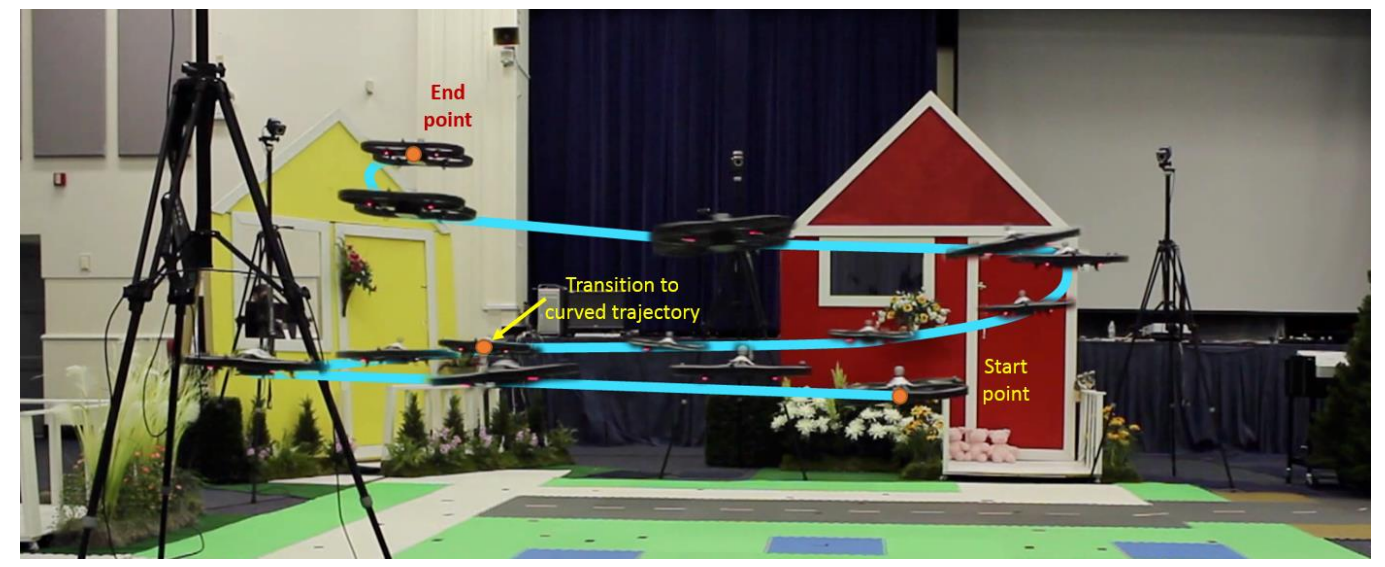

Figure 5: Single asset executes a spiral to loiter pattern for science data collection

This type of flight pattern is especially relevant to NASA for atmospheric and earth science data collection. Figure 6 shows an exemplar mission ${ }^{18,19}$ in which a geographical area of interest (AOI) has been defined and each vehicle in the mission has as assigned role in achieving the science mission.

In partnership with student interns from University of Illinois Urbana-Champaign and Carnegie Mellon University, we have developed a concept of operations for locating the source of an airborne pollutant. Give $n$ participating agents, an area of interest (AOI) is to be searched in a collaborative way and, when the source is identified, the discovering agent will communicate the location to its teammates who will replan their paths to reach the AOI and loiter around the location, serving as a beacon for humans and machines. 


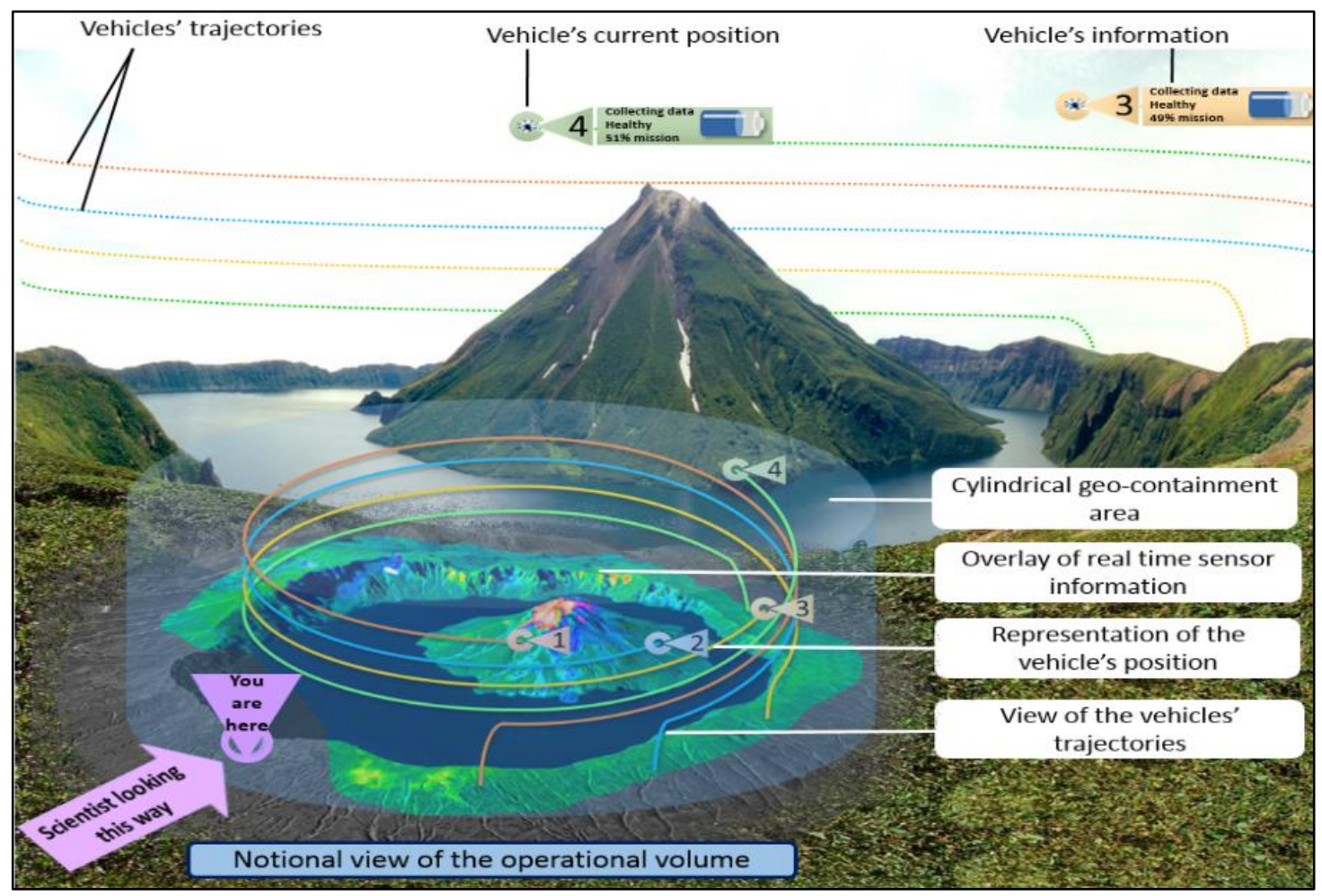

Figure 6: Exemplar Science Mission

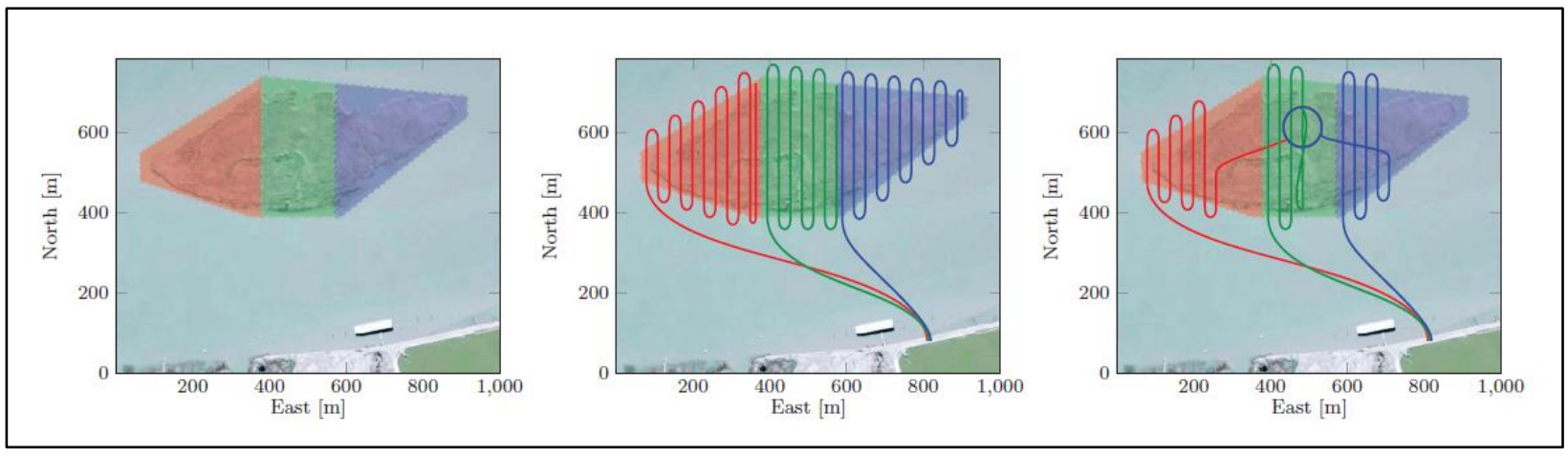

Figure 7: Left panel shows the AOI divided between the three vehicles (n=3). Middle panel shows the trajectories planned for the three vehicles to conduct the search mission. Right panel shows the replanned trajectories after the source of the pollutant has been located in the green/middle section of the AOI.

The addition of real assets in a realtime simulation environment brings with it requirements for increased communications throughput and operational space. Extending the number of participants in virtual simulation imposes no additional communications or infrastructure burden and will serve as a first step in supporting "massively multiple" participants.

\section{Conclusions}

The Autonomy Incubator is a co-located team composed of researchers from a range of discipline areas including but not limited to computer science, robotics, electrical engineering, mechanical engineering, aerospace engineering, psychology, machine vision, and machine learning. These Principal Investigators (PIs) are supported by qualified UAS pilots, flight safety personnel, range safety officers, and technicians. Building on decades of experience and 
success in the design, fabrication, and integration of safe and reliable automated systems for space and aeronautics, we are working towards a verifiable safe software architecture that supports rapid integration of software and hardware by leveraging community standards such as DDS and abstracting hardware-specific interfaces away from software system interfaces. Additionally, the AI is guiding the first generation of an on-site simulation and test environment for performance assessment of autonomous aerial systems from both inside and outside of NASA.

One of these test environments is modeled after Massively Multiplayer Online Role Playing Games in support of research and development towards an Ab Initio NAS Design. The Ab Initio test environment will transparently transition from software simulation to an indoor flight area of over 70,000 cubic feet in the Langley Autonomy and Robotics Center (home of the AI) to two types of contained outdoor flight (tethered and caged) and finally to outdoor flight in NASA LaRC's onsite Phase I Certificate of Waiver or Authorization (COA) area as shown in Figure 8.

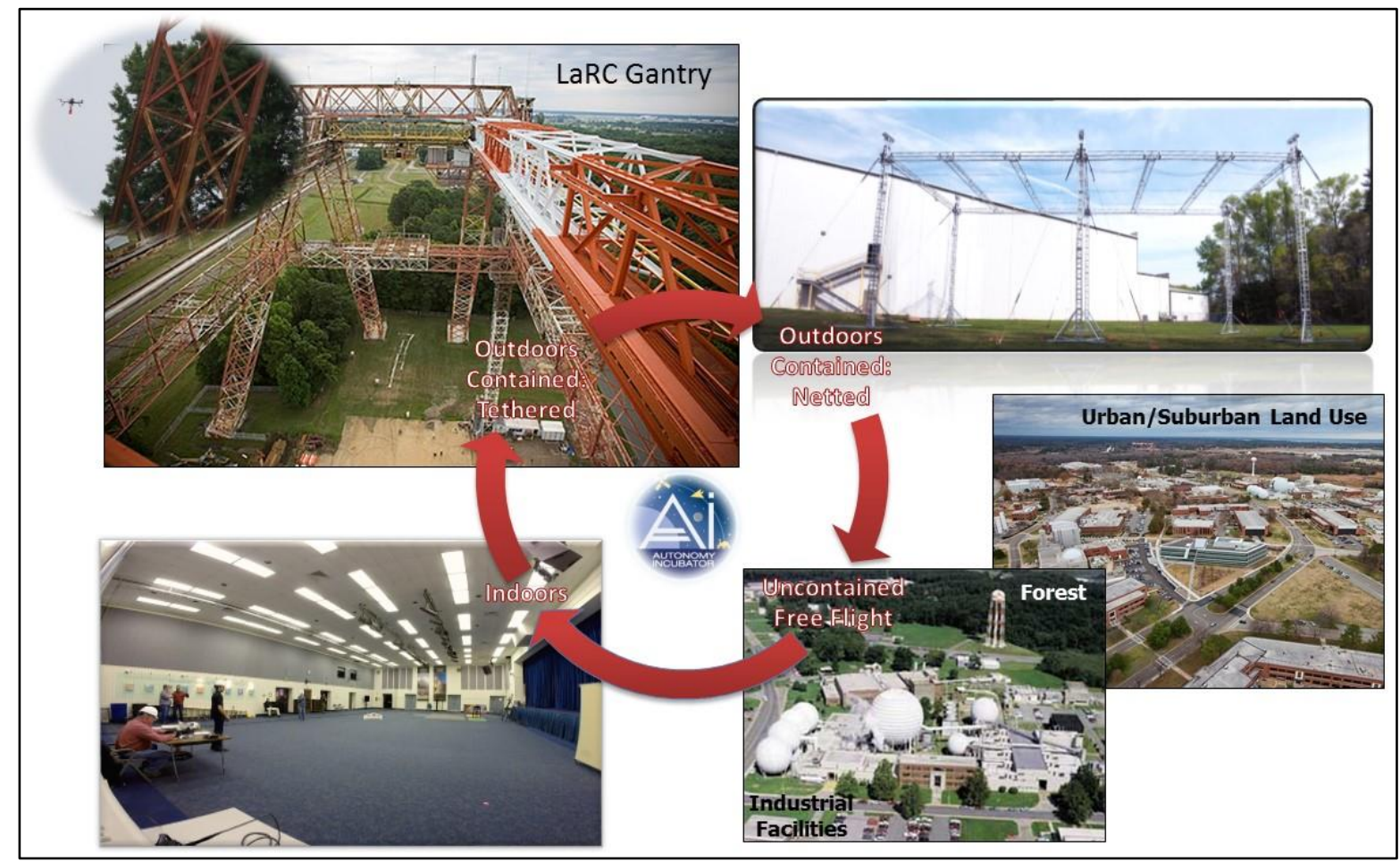

Figure 8: NASA LaRC's Operational Flight Areas

The goal is simulation of many thousands of aerial assets equipped with various levels of intelligence, capability, and behaviors. These assets will be associated with personality types that will range from collaborative to benign to destructive. Human player participants can be integrated with these machine agents in order to assess the efficacy of proposed Ab Initio NAS architectures and their sensitivities to nominal and off-nominal situations. If successful, the $\mathrm{Ab}$ Initio NAS effort will inform and enable the design of survivable aircraft to prevent degradation in safety and ensure safe and efficient access to airspace for the public and industry in the face of a massive influx of autonomous systems with a subset of non-cooperative participants can severely degrade the safety of the humans, both in the aircraft and on the ground.

\section{Acknowledgments}

This effort would not be possible with the passion and innovation of the members of the NASA LaRC Autonomy Incubator team and the unflinching support of senior management at NASA LaRC: Loc Tran, Jim Neilan, Anna Trujillo, Ralph Williams, Ben Kelley, Kyle Andrews, Carol Castle, and Vicki Crisp. Autonomy Incubator student efforts during the summers of 2014, 2015, and 2016 have also aided this work. Special thanks go to Meghan Chandarana from Carnegie Mellon University as well as Javier Puig-Navarro and Bilal Mehdi from University of Illinois Urbana- Champaign along with their advisor, Naira Hovakimyan. 


\section{References}

[1] B. D. Allen, Interviewee, NASA Autonomy Incubator Pushing for Intelligent Machines. [Interview]. 16 April 2014, https://www.youtube.com/watch?v=6udRw7ltDtA.

[2] Boyd, John, R., The Essence of Winning and Losing, http://www.danford.net/boyd/essence.htm, 28 June 1995

[3] Allen, D., Cross, C., Tran, L Motter, M., Neilan, J., Qualls, G., Rothhaar, P., Trujillo, "Who's Got the Bridge? Towards Safe, Robust Autonomous Operations at NASA Langley's Autonomy Incubator", AIAA Aviation 2015, Dallas, TX, 2015, AIAA 2015-2897.

[4] Natalia M. Alexandrov and Thomas A. Ozoroski, "Design for Survivability: An Approach to Assured Autonomy", AIAA Aviation 2016, Washington DC, June 2016.

[5] Unmanned Aircraft System (UAS) Traffic Management (UTM), http://utm.arc.nasa.gov/index.shtml

[6] Lewin, R., Complexity: Life on the edge of chaos, University of Chicago Press, 2nd edition, February 15, 2000

[7] Nicolis G. and Prigogine I., Exploring complexity. St. Martin's Press; 1st Edition, October 1, 1989

[8] https://www.flickr.com/photos/benimoto/2775801876 [creative commons license] https://www.youtube.com/watch?v=1VhYRnQZv5c [creative commons license] https://www.flickr.com/photos/juanpol/8232592 [creative commons license]

[9] https://www.youtube.com/watch?v=wdXpkx7u39o [creative commons license]

[10] https://www.youtube.com/watch?v=1CIGKAnxiXY [creative commons license]

[11] Bartle, Richard (2003). Designing Virtual Worlds. New Riders. ISBN 0-13-101816-7.

[12] "Data-Distribution Service Portal," [Online]. Available: http://portals.omg.org/dds/. [Accessed 21 May 2015].

[13] C. D. Cross, M. A. Motter, H. J. Neilan, G. D. Qualls, P. M. Rothhaar, L. Tran, A. C. Trujillo and B. D. Allen, "Towards an Open, Distributed Software Architecture for UXS Operations," AIAA Aviation 2015, Dallas, TX, 2015.

[14] ROS, [Online]. Available: http://www.ros.org/. [Accessed 21 May 2015].

[15] https://en.wikipedia.org/wiki/Alignment_\%28Dungeons_\%26_Dragons\%29

[16] Husni Idris, Daniel Delahaye, David Wing. Distributed Trajectory Flexibility Preservation for Traffic Complexity Mitigation. ATM 2009, 8th USA/Europe Air Traffic Management Research and Development Seminar, June 2009, Napa CA.

[17] Natalia Alexandrov. "Control of Future Air Traffic Systems via Complexity Bound Management", 2013 Aviation Technology, Integration, and Operations (ATIO) Conference, AIAA Aviation, August 2013, Los Angeles, CA, AIAA 2013-4419.

[18] S. Bilal Mehdi, Javier Puig-Navarro, Ronald Choe, Venanzio Cichella, Naira Hovakimyan, Meghan Chandarana, Anna Trujillo, Paul M. Rothhaar, Loc Tran, James H. Neilan, and B. Danette Allen, "A Safe Cooperative Framework for Atmospheric Science Missions with Multiple Heterogeneous UAS using Piecewise Bezier Curves", Aviation 2016, Washington DC, June 2016.

[19] M. Chandarana, A. Trujillo, K. Shimada and B. D. Allen, "A Natural Interaction Interface for UAVs Using Intuitive Gesture Recognition", 7th International Conference on Applied Human Factors and Ergonomics, July 2016, Orlando FL.

[20] Sky for All: Air Mobility for 2035 and Beyond, https://herox.com/SkyForAll 